\title{
Environmental Scanning Electron Microscopic Study Of Electron Impacted High Explosive Materials
}

\author{
S.K.Haita, ${ }^{*}$, B.G. Polke ${ }^{a}$, S. Venugopalan ${ }^{a}$, B.R. Gandhe ${ }^{a}$ \& A.S. Rao ${ }^{a}$ \\ aHigh Energy materials research Laboratory, Sutarwadi, P.O. Armament, Pune, India \\ 411021
}

An environmental scanning electron microscope (ESEM) has been used to reveal detonation behavior in highly insensitive explosives through electron impact study. The high explosive materials RDX, HMX, HNIW and TATB show the stability behavior in line with thermal stability. Thermal behavior of these explosives are compared with morphological observations in solid to gas phase transitions. Understanding of knowledge of reaction sites and evolution of hot spots generation can lead to improvements in the safety aspects of explosive handling as well as in the study of futuristic warfare by protecting warheads of the missile on flight from perturbing high voltage radiation. The behavior of these explosive compounds is pictured to understand the initiation, subsequent structure deformation, loss of surface periodicity and detonation.

The samples of RDX, HNIW, HMX and TATB are bombarded by high-energy electron beam. Generally on exposure to these high-energy particles the sample surfaces may get irreversibly heated at a high rate and finally detonated. Electron micrographs of RDX, HNIW, HMX and TATB are presented in figure 1,2,3 and 4 respectively. It was found from the electron microscopy that samples with higher thermal stability tend to be more resistant to electron beam damage. Similar observation for non-energetic organic compounds was also reported [1]. The detonation behavior is propagated by generation of activated reaction spots, which is shown for RDX. At reaction sites craters are resulted, the larger ones showed hemispherical shape, whereas the smaller ones as swollen craters. The craters showed rounded edges with increasing diameter. These reaction sites were in the shape of hillocks; a closer look of the uneven shape indicates that they have arisen from the effect of electron impact subsequently by melting, internal reaction and ballooning. The base of these hillocks is about 10 times larger in diameter. The volume of the swelled crystal increases considerably, due to solid to liquid and immediately to gas phase conversion during ballooning. These supports the idea that gas generation is taking place. Large volcano like hillocks with liquid being sputtered is also reported in literature [2]. In all crystal surfaces a large variety of defects exist, among them a few are critical for detonations. Kinetic energy supplied by the electron beam starts the reaction in microscopically small sites, some of which can precariously develop into hot spots and then cause violent reaction.

It is found that monocyclic non-aromatic nitramines i.e. RDX and HMX show strong melting behavior before decomposition, HNIW and TATB decomposes without melting. However, the crystals of HNIW slowly coalesce indicating the process of melting commences. The cracked crystal surface of TATB on prolonged perturbation by electron beam does not tend to form dark spots, whereas in the case of HMX small spots are generated. TATB beamed for 6 hours at 30 $\mathrm{kV}$, had shown neither melting nor dark spot generation, being the most thermally and electronically insensitive munition. A comparison of their thermal and electron impact behavior is presented in table 1 .

References

1. Kumar, S. W, \& Adams, W. (1990) Polymer 31, 15-22.

2. Sharma, J. \& Beard, B.C. (1991) technical report, NAVSWC TR 91-682, naval Surface warfare centre, Silver spring, MD. 


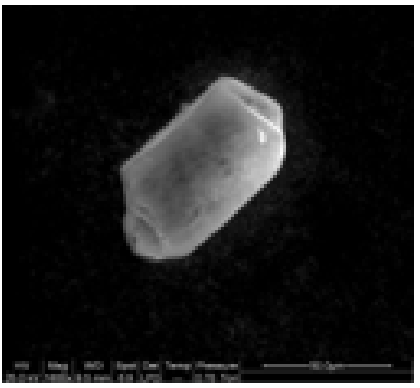

Fig, 1A.bmp

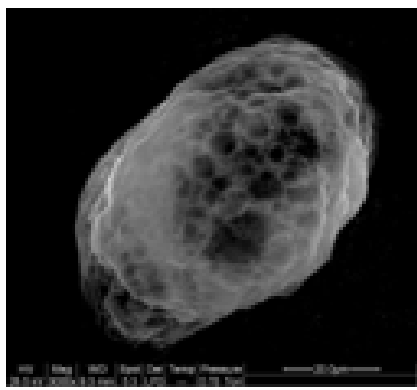

Fig. 1E.bmp

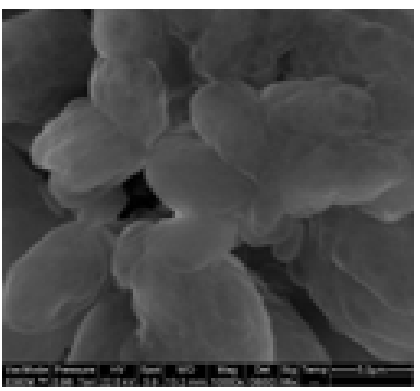

Fig, 2D.bmp

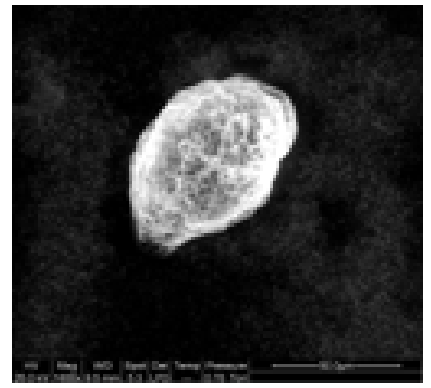

Fig. 1B.bmp

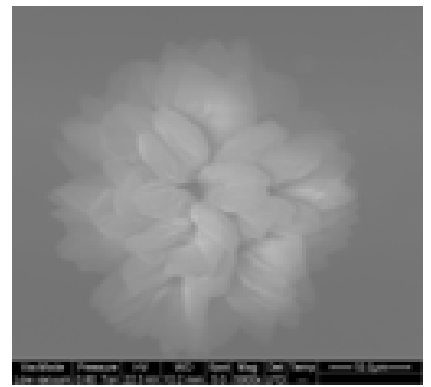

Fig. 2A.bmp

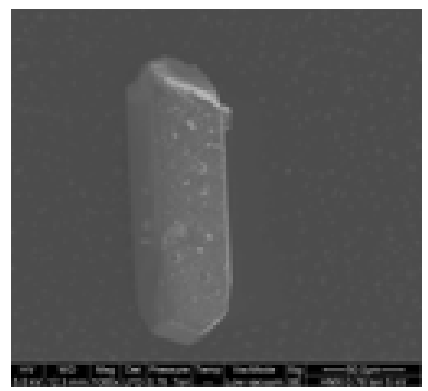

Fig. 3A.bmp

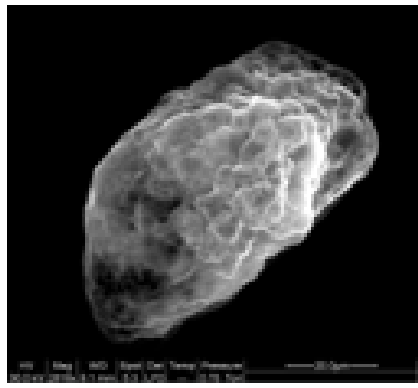

Fig. 1C.bmp

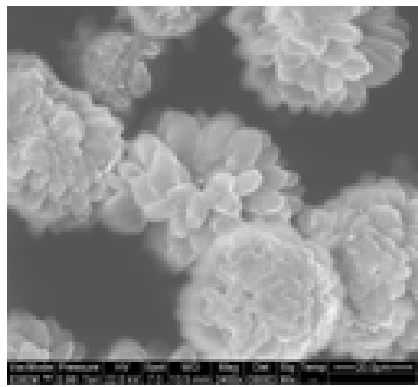

Fig. 2B.bmp

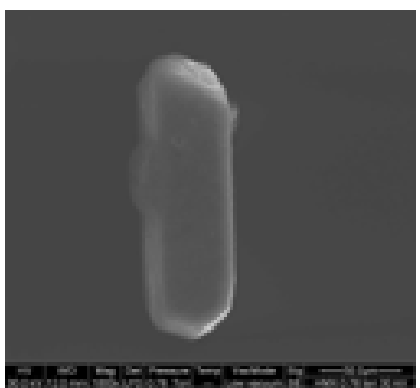

Fig. 3B.bmp

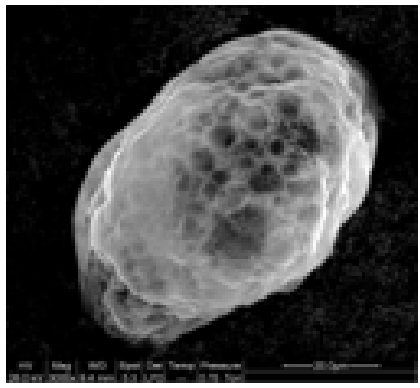

Fig, 1D.bmp

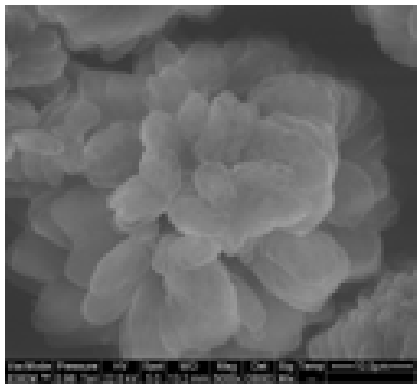

Fig. 2C.bmp

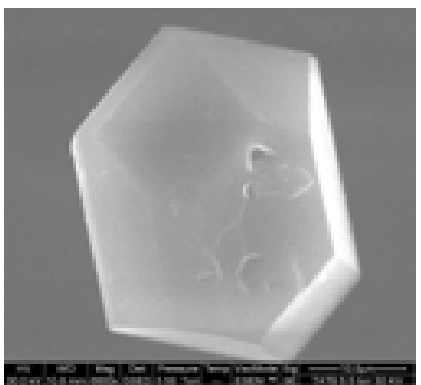

Fig 4.bmp

TAELE 1

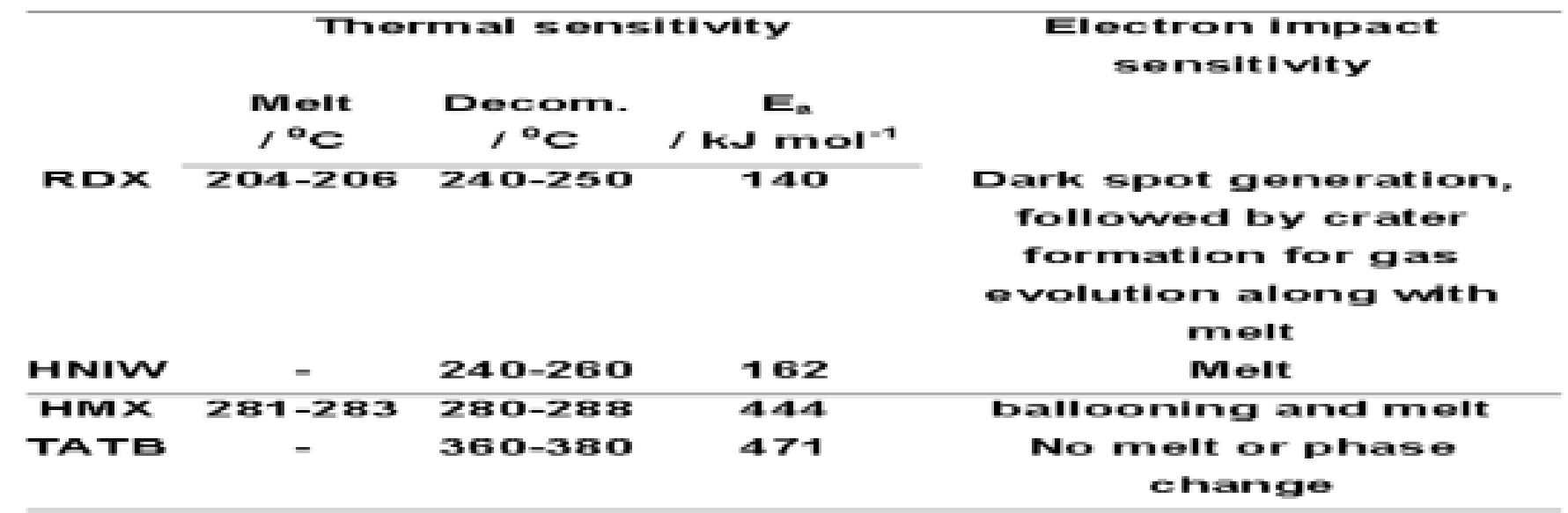

